\title{
Kinetic development of crystallization fronts in complex plasmas
}

\author{
M. RUBIN-ZUZIC ${ }^{1 *}$, G. E. MORFILL ${ }^{1}$, A. V. IVLEV ${ }^{1}$, R. POMPL ${ }^{1}$, B. A. KLUMOV ${ }^{1}$, W. BUNK ${ }^{1}$, \\ H. M. THOMAS ${ }^{1}$, H. ROTHERMEL ${ }^{1}$, 0. HAVNES ${ }^{2}$ AND A. FOUQUÉT ${ }^{3}$ \\ ${ }^{1}$ Max-Planck-Institute for Extraterrestrial Physics, 85741 Garching, Germany \\ ${ }^{2}$ Department of Physics, University of Troms $\emptyset$, 9037 Troms $\emptyset$, Norway \\ ${ }^{3}$ Université d'Orléans, 45067 Orléans, France \\ *e-mail: zuzic@mpe.mpg.de
}

1 lthough it is relatively straightforward to measure the atomic structure of crystals, the detailed dynamics of crystal growth (including the evolution of self-organization, structure formation, and the associated kinetic and thermodynamic development) remains one of the most important topics of solid-state physics ${ }^{1-3}$. Very little is known, for instance, about the evolutionary paths of crystal structure development, and the structural complexity of the surface down to atomic dimensions and its change with time. This is, to a large extent, due to the lack of suitable systems that can be studied at the appropriate length- and timescales. Here, we report experimental observations of a crystallization front propagating in a supercooled region of a three-dimensional fluid complex plasma. The structure and evolution of the front are analysed using kinetic measurements of individual microparticle dynamics. It is found that the fluid-crystalline transition is accompanied by short-lived 'nano'-crystallites in the fluid and 'nano'-droplets in the crystalline phases, that the surface structure is scale-free (fractal) in the experimentally accessible regime (2-10 lattice distances) and that crystal growth follows a universal self-organization pattern at the particle level, leading to oscillations in the surface roughness.

Crystal growth may take place under a variety of conditions, the most important parameters being the flux of new particles (atoms) onto the existing surface, the cooling process and associated surface attachment, the degree of undercooling, and the latent energy. This may result in various types of growth, for example, amorphous, polymorphous, epitaxial, dendritic, and the formation of diverse structures. The situation is complicated further by the possible existence of anisotropies, instabilities, stochastic noise, and so on, and their effects on the self-organization that is the basic signature of crystallization. If there is a transition region between the crystalline and fluid phases, its width presumably depends on the cooling-time (length) scale, which in turn also relates to the latent heat released at the interface. The surface structure could then become quite complex.
Understanding all of these influences is, of course, important for materials design. In particular, it has become clear that nanoscale structures and properties can have a profound effect on the optical, electrical and mechanical properties of the meso- or macroscopic material. Therefore, kinetic measurements (at the individual particle level) of the crystallization process and its dynamics are of great interest. Complex plasmas (electrons, ions, neutral gas and charged microparticles) are ideal systems for such measurements: they are optically thin, charge-neutral, weakly damped systems, where the dominant component, the microparticles, can be visualized individually ${ }^{4,5}$.

The principal aim of experiments with complex plasmas, as well as with colloidal suspensions, is to pinpoint generic features and processes, and to study them under conditions not previously accessible. In the case of complex plasmas, this has a twofold significance. First, it enhances our knowledge about these unusual states of soft matter (the crystalline and fluid complex plasmas) and second, it provides invaluable insights into possible universal processes, hierarchies and scaling properties-complementing colloidal suspension studies, which can serve as good models for atomic/molecular materials ${ }^{6,7}$.

Colloidal systems are strongly overdamped (fluid density $\sim 1 \mathrm{~g} \mathrm{~cm}^{-3}$ ), whereas complex plasmas experience much less damping (neutral gas density $\sim 10^{-6} \mathrm{~g} \mathrm{~cm}^{-3}$ ). This means that physical processes up to the natural frequencies (dust plasma frequency $\sim 10^{1}-10^{2} \mathrm{~s}^{-1}$ ) act essentially undamped in complex plasmas and, hence, these systems behave much more like 'one-phase' systems ${ }^{8}$.

In this paper, we present and discuss the first experimental observations of the propagation of a crystallization front through an undercooled fluid complex plasma. We investigate the dynamical evolution of the surface structure at the kinetic level, and determine its micro-roughness down to scales of individual grain separations.

Examples from the measurements are given in Fig. 1, where the crystallization progress is shown at two different times. Freezing starts at the bottom, then the crystallization front moves up 

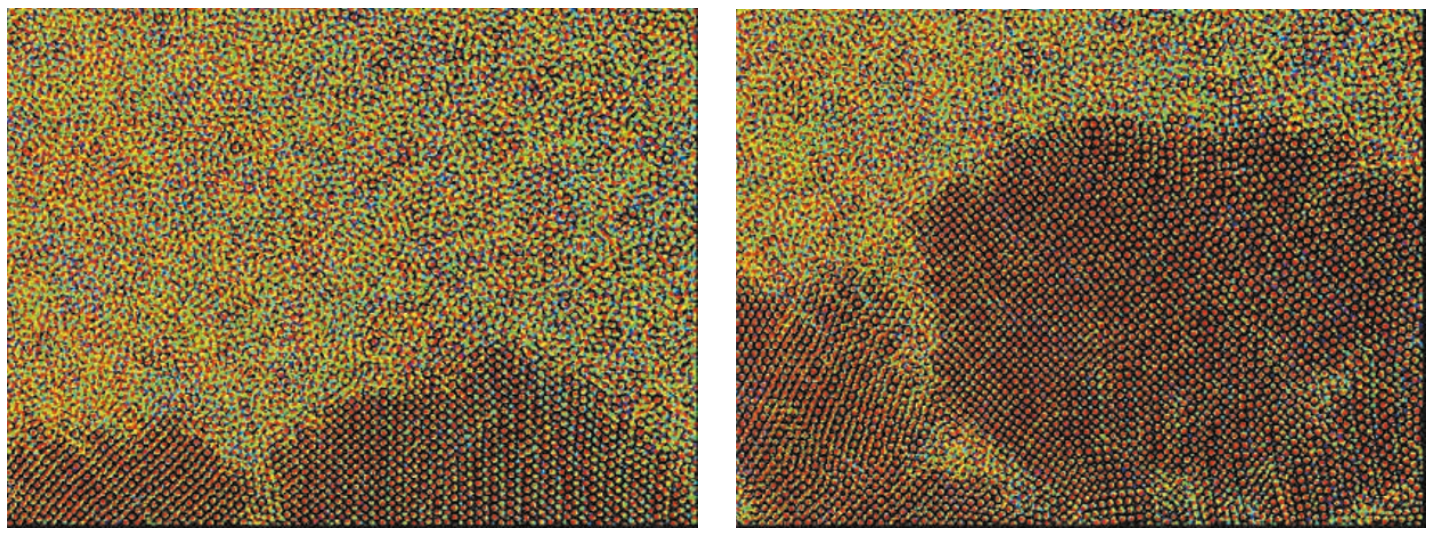

Figure 1 Propagation of the crystallization front. The images $(6.4 \mathrm{~mm} \times 4.8 \mathrm{~mm})$ show the development of the front at two different times ( $\approx 16 \mathrm{~s}$ apart). Each image is a superposition of ten consecutive video frames ( $0.67 \mathrm{~s}$ in total) colour-coded from blue (through green and yellow) to red. In the fluid regime (left), where the diffusive particle motion is well pronounced, a more or less homogeneous mix of all colours is seen. In the crystalline regime (right), where the (average) particle positions are fixed, the last colour used (red) hides the others and hence dominates, leading to a clear visual identification of ordered crystalline structures. Globally, the crystalline region consists of two domains of different lattice orientation (or structure), with the 'interfacial melting' in between.

through the system. The crystalline and fluid phases are easily distinguishable. In the former, diffusion is inhibited and particles mostly exhibit caged motion, thus forming well-ordered structures, whereas in the latter the diffusive motion is clearly seen.

The analysis of the crystallization process was carried out as follows (technical details are presented in the Methods section).

First, to distinguish the fluid and solid regimes, we used an 'overlapped image' algorithm. As the measure of the local diffusive motion, we calculated a 'displacement area' for individual particles during a few frames (no motion corresponds to the zero displacement area). Figure 2 shows the colour-coded map of the local displacement area, which decreases by a factor of $\approx 2.1$ (on average) on transition from the fluid to the crystalline regime. This technique allows us to distinguish the boundaries with fairly high spatial precision (by varying the threshold value of the displacement area, we obtained an uncertainty of about two interparticle distances). The calculated vertical velocity of the crystallization front (averaged over the front) is about one interparticle distance per second. Note that the overlapped-image algorithm allows us to quantify the solidification in terms of the mean (namely, diffusive) particle displacement in the fluid phase. Therefore, the proposed method is (somewhat) complementary to the Lindemann criterion of melting.

Then, we calculated the local particle number density. In the crystalline regime, the temporal variation of the density was insignificant over the entire observation time (less than $1 \%$ during about $40 \mathrm{~s}$ ). Vertically, the density decreased by about $6 \%$ over $\approx 4 \mathrm{~mm}$ height (presumably due to gravity). This corresponds to a characteristic vertical inhomogeneity scale of $\sim 10 \mathrm{~cm}$, which is $\sim 10^{3}$ times larger than the mean interparticle distance. Thus, in terms of the density, the observed crystal was remarkably homogeneous. The average density in the crystalline region was $2,400 \pm 100 \mathrm{~mm}^{-3}$, which yields a mean interparticle distance $\Delta \approx 75 \mu \mathrm{m}$.

The observed growth of the crystallization front seems to suggest some equilibrium between small structure formation and destruction. For a purely dendritic (namely, diffusion-limited) growth, we would expect the more exposed regions to have a higher probability of particle attachment, so that convex structures should grow. In the opposite limit, where the cooling of the liquidus is achieved fastest for particles that have a larger number of attachment points, small concave regions are favoured, with the logical extreme being epitaxial growth by single layers and planar, aligned crystal fronts 9 .

As a quantitative measure of the growth, we calculated an estimator of the fractal dimension for the observed two-dimensional (2D) contour of the crystallization front (Fig. 3). The results show that the front can indeed be characterized by a fractal dimension with the average value $\langle D\rangle \approx 1.19$. Moreover, we see that the fractal dimension develops an oscillatory behaviour, $1.16 \leq D(t) \leq 1.21$. Note that by varying the threshold value of the displacement area, we observed that the crystallization front shifted slightly up or down as a whole, thus keeping the fractal dimension almost unchanged.

The temporal fluctuations of the fractal dimension imply that the surface roughness increases (fractal dimension larger) then decreases (smaller), which is clearly illustrated in the inset of Fig. 3. Using the derived values of the timescale of the oscillations, $\approx 1.4 \mathrm{~s}$, and the average growth rate, $\approx 1 \Delta \mathrm{s}^{-1}$, we conclude that approximately one layer is deposited per fluctuation period.

The calculated value of the fractal dimension allows us to characterize the growth type. Typical dendritic structures are known $^{10}$ to have $D \approx 1.42$, whereas the planar epitaxial growth yields unity as the obvious lower limit. Hence, in terms of the calculated fractal dimension, the observed crystallization falls in between the two growth extremes. The fractal law holds down to the smallest scales of $2 \Delta$. The largest scale we can consider within our observational constraints is $10 \Delta$. A power-law dependence (namely, fractal structure) implies that there is no length scale that can be defined as significant for the growth process in this size regime, and the growth appears to be universal.

Let us discuss peculiarities of the fluid-crystalline transition in detail. Figure 2 shows that the overlapped-image analysis of the particle motion provides a robust and precise determination of the crystallization front. Surprisingly, it also reveals the existence of small 'temperature islands' - transient 'cold' crystallites (inside the fluid regime) and 'hot' droplets (inside the crystalline regime) whose lifetimes depend on their size (see insets in Fig. 2). Typical crystallites contain $30-150$ particles, and droplets contain 50-250 particles.

The evolution of the crystallites can be understood in terms of the thermodynamics ${ }^{8,11-13}$. Random fluctuations may produce local 


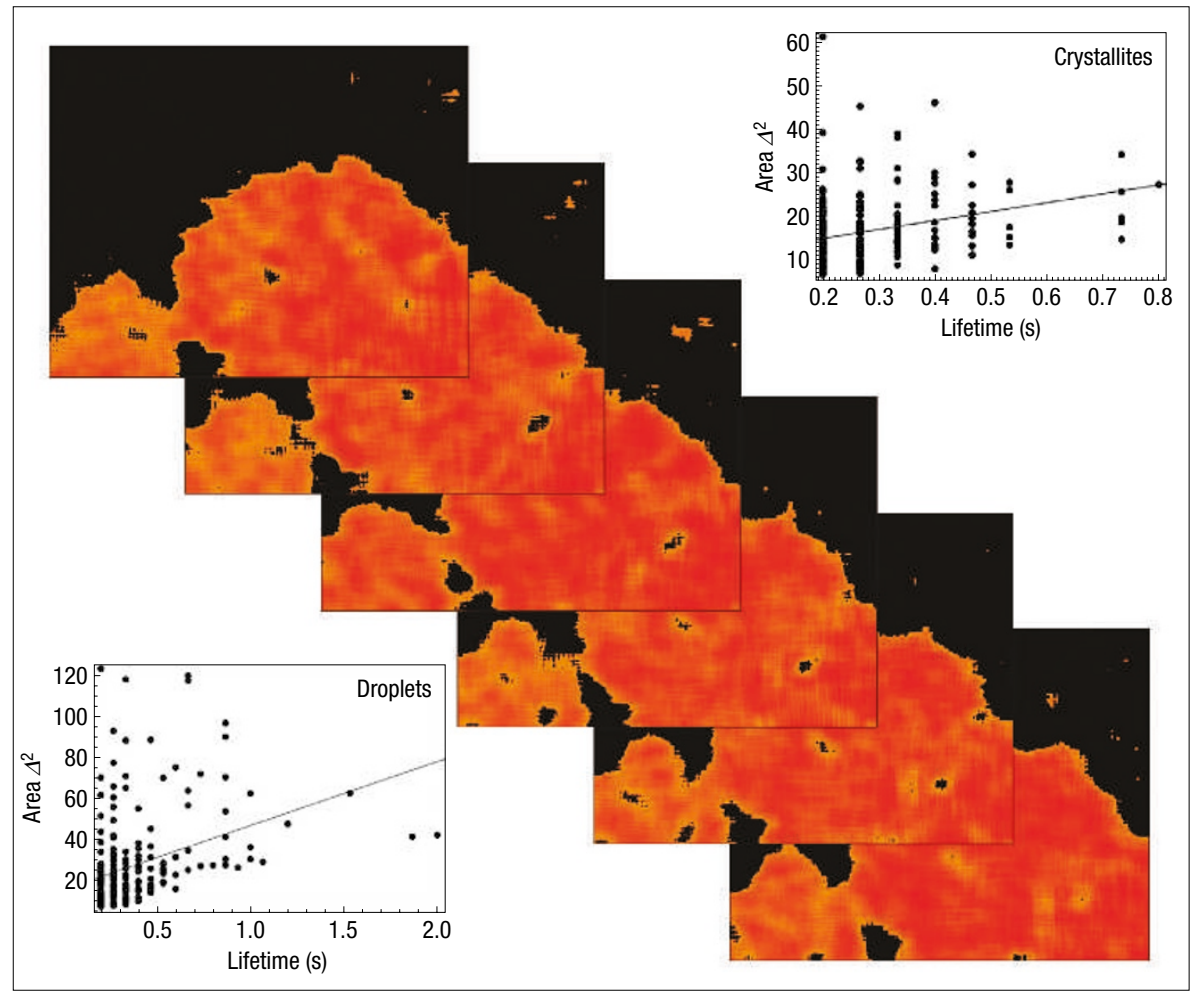

Figure 2 Series of colour-coded images of the crystallization process. Consecutive images are $1 / 15 \mathrm{~s}$ apart. The colour represents the particle 'displacement area' $\delta A$ (see Methods): red implies high crystalline order (low $\delta A$ ), black denotes the fluid phase (high $\delta A$ ), and yellow indicates transitional regions. In the fluid phase, $\delta A$ is, on average, $\approx 2.1$ times larger than in the crystalline regime. Along with the crystallization front propagating upwards, droplets and crystallites are seen that may grow and then dissolve again. The insets show that their lifetimes increase with area size (measured in units of squared interparticle distance, $\Delta^{2}$ ). The spontaneous growth and disappearance of crystallites in the fluid regime and droplets in the crystal regime, near the propagating front, are one of the new insights from our study.

temperature decreases, which, in turn, induce the formation of seed crystallites. However, if the size of a given crystallite is smaller than the critical (nucleation) threshold, the resulting decrease of the bulk free energy is compensated by an increase in the surface energy, that is, the surface tension exceeds the internal pressure. Then the crystallite will shrink, and eventually disappear. Naturally, larger crystallites produced by a stronger local perturbation will survive longer. The upper inset in Fig. 2 clearly demonstrates this trend. Note that many large crystallites (presumably above the nucleation threshold) may have relatively short lifetimes, because they are absorbed by the propagating crystallization front.

At first sight, the droplets observed in the crystalline regime present an enigma. The mechanism responsible for their formation must be quite different, because thermodynamically, both the bulk and the surface contributions cause the free energy to increase, as in the case of bubbles in boiling water. We propose the following mechanism. Experiments and computer simulations suggest that the nucleation usually passes through a certain metastable phase. For instance, experiments with colloidal suspensions ${ }^{8,11,14,15}$, as well as the corresponding numerical simulations ${ }^{11,16}$, have shown the existence of a metastable random hexagonal close-packed (h.c.p.) phase (mixture of h.c.p. and face-centred cubic (f.c.c.)-like lattice planes with hexagonal order). This implies that after the initial solidification, a gradual relaxation to a ground state (for example, body-centred cubic or f.c.c. lattice domains) occurs downstream from the crystallization front. This is naturally accompanied by a release of latent heat. We believe that the observed 'hot' droplets are a local manifestation of this relaxation. The larger the droplet, the longer it takes to dissipate the released heat, and the longer its lifetime.

Presumably, both mechanisms described above (for the crystallite and droplet formation) may operate in any medium undergoing non-equilibrium crystallization. This suggests that our findings may represent generic kinetic properties of solidification in undercooled liquids. The discovery of the droplets (or local excited regions) could be important in the context of annealing processes and technology. Of course, further experiments, covering other parameters, are necessary to obtain a comprehensive picture of the (nano) droplet kinetics.

It is noteworthy that the growing crystal exhibits two large domains, which are distinguishable in Fig. 1 by the lattice orientation (and possibly also by the lattice structure, although with a $2 \mathrm{D}$ slice measurement this is not a unique option). The two domains are separated by an interface of typical width $\sim 3 \Delta$, which appears to be 'molten' in terms of the kinetic energy (see Fig. 2), but at the same time maintains some structural order. As with the observed droplets, we suggest that this feature, 'interfacial melting, is also a consequence of the relaxation from an excited to a more stable lattice type.

Finally, to analyse the crystallization processes further we carried out molecular dynamics simulations. Similar to the experiment, the crystallization front in the simulations always starts from the lower boundary and propagates upwards. We detect dust acoustic waves excited at the front, which transport the latent heat away. It is remarkable that even in the $2 \mathrm{D}$ case, the distribution of the local kinetic energy exhibits many of the important features 


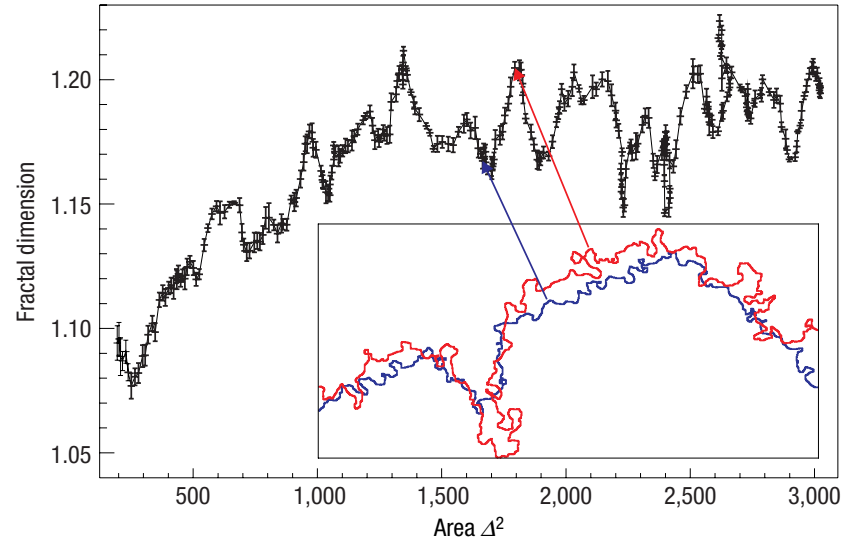

Figure 3 Fractal structure of the crystallization front. The plot shows how the fractal dimension of the 2D contour of the front evolves during the growth phase, with respect to the crystallization area (measured in units of $\Delta^{2}$ ). The error bars are the bootstrap estimates ${ }^{21}$ for the standard deviation. Every increase in area is accompanied by a characteristic oscillation in the fractal dimension, implying a growth of surface complexity followed by a smoothing of surface structures. This is illustrated in the inset with two typical contours of the crystallization front representing two snapshots (at different times corresponding to a maximum and a minimum of the fractal dimension) of the boundary between the fluid and crystalline phases.

seen in the experimental data, such as the complex structure of the front and the temperature islands (see Fig. 4). However, the quantitative agreement between experiment and simulations, in terms of the front speed, can only be obtained with full 3D simulations (see Fig. 4). A parameter study then shows that in order to obtain a match with the growth velocity observed in the experiment, particle charges in the range $(2-4) \times 10^{3} e$ are required, whereas the dependence on the screening (Yukawa) length is quite weak.

The crucial influence of the dimensionality on the crystallization kinetics, discovered through the molecular dynamics simulations, is fully consistent with the experimental investigations. It has been shown ${ }^{17}$ that the temperature relaxation processes in strongly coupled (solid) systems are slowed down dramatically compared to weakly coupled systems, because the probability of a transition from one to another (metastable) solid state decreases exponentially as the particles cool down ${ }^{18}$. Going from 2D to 3D systems increases the number of possible metastable states, which explains why the crystallization takes longer for the 'higher dimensional' case.

The local order analysis, for the 3D simulations at the initial stage of crystallization, with an algorithm similar to that used in ref. 7, yields the result that only a few per cent of the particles in the crystalline regime organize themselves in a (presumably ground-state) f.c.c. lattice. Most particles in the vicinity of the front, form the metastable h.c.p. phase. Thus, the molecular dynamics simulations support the hypothesis of the 'hot' droplet formation and the interfacial melting through a metastable phase, which was suggested here to explain the experimental observations.

\section{METHODS}

\section{EXPERIMENT}

Initially, a large plasma crystal (containing $\sim 10^{7}$ melamine formaldehyde particles of diameter $1.28 \mu \mathrm{m}$, with a size dispersion of $\pm 0.056 \mu \mathrm{m}$ ) was formed in a radiofrequency discharge plasma (at $13.6 \mathrm{MHz}$ frequency and

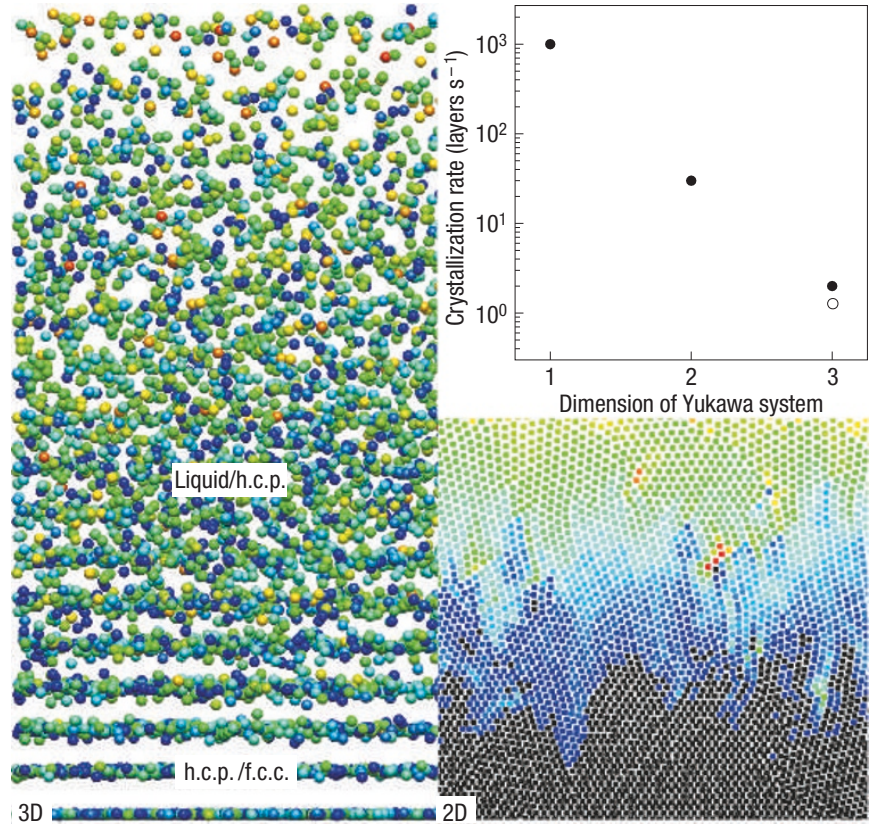

Figure 4 Molecular dynamics simulations of the crystallization front. The figure shows the side-view snapshot of the velocity field and particle positions in 2D and $3 \mathrm{D}$ Yukawa systems. The velocity magnitude is colour-coded. It rises from black (room temperature) to yellow by a factor of four. The front has a complex structure, with a transition layer and transient 'temperature islands', which is clearly seen even for the 2D case. In the 3D simulations, the crystallized particles mostly form the metastable h.c.p. lattice, with just a few per cent of f.c.c. The plot shows the dependence of the crystallization front velocity on the dimensionality (solid points). It reveals the crucial role of the dimensionality in strongly coupled systems, only the $3 \mathrm{D}$ simulations provide quantitative agreement with the experimental data (open circle).

0.23 mbar Ar gas pressure). The plasma crystal was then melted down by a sudden power decrease, and subsequently allowed to re-freeze.

The crystal was illuminated by a vertical laser sheet, and recorded from the side with a CCD camera (pixel size $6 \mu \mathrm{m}$ ) at 15 fps frame rate. The selected CCD camera type provides a reasonable trade-off between resolution (both temporal and spatial) and technical limitations. It is necessary to have a sufficiently large field of view, and to record over a sufficiently long time to observe large-scale structures and long-term evolution of the crystallization front.

\section{OVERLAPPED-IMAGEALGORITHM}

A standard image-analysis procedure ${ }^{19}$ was implemented to identify individual particles in each video frame. Due to overexposure, sub-pixel-sized particles occupied $\geq 10$ image pixels, and therefore every single particle could be identified and its position could be determined with sub-pixel accuracy. The area of the $i$ th particle image in the $j$ th frame was defined as $A_{i j}$. A short series of consecutive frames were superimposed to form overlapped images.

Subtraction of the initial area yielded the 'displacement area' during $\delta j$ frames, $\delta A_{i}(\delta j)=\bigcup_{j}^{j+\delta j} A_{i j}-A_{i j}$ (where $\bigcup$ is the union operator). Transition from the fluid to the solid regime is characterized by an abrupt decrease of $\delta A_{i}$. The ratio of average values of $\delta A_{i}$ (for $\delta j=4$ ) in the fluid and solid regions is $\approx 2.1$. These values are calculated with an accuracy (relative standard deviation) of $\approx 2 \%$ and $4 \%$, respectively. The half-width of the transition region is $\approx 1.9 \Delta$, with $\approx 18 \%$ standard deviation.

\section{DETERMINATION OF LOCAL DENSITY}

The transverse intensity distribution in the illuminating laser sheet had a gaussian profile, $\propto \exp \left(-z^{2} / \sigma^{2}\right)$ with $\sigma \approx 25 \mu \mathrm{m}$ (where coordinate $z$ is perpendicular to the field of view). By analysing individual video frames, the 
threshold intensity of the particle identification was derived, which corresponded to the particle slab that was $85 \pm 5 \mu \mathrm{m}$ thick. This allowed us to directly determine the local number density in the crystalline region, irrespective of the particular structure. In the fluid regime, unfortunately, the rapid diffusive motion caused a substantial fraction of the detected particles to leave and (probably) re-enter the illuminated field of view, thus making the described technique unreliable.

\section{CALCULATION OF FRACTAL DIMENSION}

An estimator of the fractal dimension for the crystallization front contour was calculated following the suggestion by Flook ${ }^{20}$ (known as the Minkowski fractal dimension). Owing to its efficiency and robustness, this method is widely used for the characterization of the fractal dimension of objects in digital images. It uses dilation logic for evaluating the increase of the object size (contour length $l$ ) with increasing spatial resolution $\left(\propto r^{-1}\right)$. The scaling range between $r_{\min }=2 \Delta(\approx 14$ pixels $)$ and $r_{\max }=10 \Delta(\approx 67$ pixels $)$ was chosen for the polynomial least-square fit in the $\log l$ versus $\log r$ diagram. The error bars given in Fig. 3 are the bootstrap estimates ${ }^{21}$ for the standard deviation of the fit. One can see that the uncertainty is much smaller than the observed oscillation amplitude of the fractal dimension. To rule out artefacts from the methodological approach, a traditional box-counting algorithm ${ }^{10}$ has also been tested to calculate the fractal dimension. The deviations between these two methods are in the range of the errors shown in Fig. 3.

\section{NUMERICAL SIMULATIONS}

The co-operative behaviour in complex plasmas is due to electrostatic coupling between the charged particles, which are believed to interact through a Yukawa-type potential ${ }^{22}$. Using parameters similar to those of the experiment, including electrostatic collisions, gravity, neutral (Epstein) drag and neutral collision-induced Langevin forces, 1D, 2D and 3D molecular dynamics simulations were carried out with 300, 8,100 and 27,000 Yukawa-interacting particles, respectively. The boundary conditions were: a potential well at the bottom (to model the sheath region at the lower radio-frequency electrode), a free boundary at the top, and periodic vertical boundaries. An initial crystallized state was melted by increasing the particle velocities suddenly, and randomly, by a factor of three.

Received 6 November 2005; accepted 27 January 2006; published 26 February 2006.
References

1. Herlach, D. M., Egry, I., Baeri, P. \& Spaepen, F. (eds) Undercooled metallic melts: properties, solidification and metastable phases. Mater. Sci. Eng. A 178, 1-313 (1994).

2. Levi, A. C. \& Kotrla, M. Theory and simulation of crystal growth. J. Phys. Condens. Matter 9 , 299-344 (1997).

3. Palberg, T. Crystallization kinetics of repulsive colloidal spheres. J. Phys. Condens. Matter 11 R323-R360 (1999).

4. Thomas, H. M. et al. Plasma crystal: Coulomb crystallization in a dusty plasma. Phys. Rev. Lett. 73, 652-655 (1994).

5. Thomas, H. M. \& Morfill, G. E. Melting dynamics of a plasma crystal. Nature 379, 806-809 (1996).

6. Pusey, P. N. in Liquids, Freezing and the Glass Transition (eds Leveesque, D., Hansen, J. P. \& Zinn-Justin, J.) 763-942 (Elsevier, Amsterdam, 1991).

7. Gasser, U., Weeks, E. R., Schofield, A., Pusey, P. N. \& Weitz, D. A. Real-space imaging of nucleation and growth in colloidal crystallization. Science 292, 258-262 (2001).

8. Khrapak, S. A., Ivlev, A. V. \& Morfill, G. E. Momentum transfer in complex plasmas. Phys. Rev. E 70, 056405 (2004).

9. Hoogenboom, J. P., Yethiraj, A., van Langen-Suurling, A. K., Romijn, J. \& Blaaderen, A. Epitaxial crystal growth of charged colloids. Phys. Rev. Lett. 89, 256104 (2002).

10. Bisang, U. \& Bilgram, J. H. Shape of the tip and the formation of sidebranches of xenon dendrites. Phys. Rev. E 54, 5309-5326 (1996).

11. Anderson, V. J. \& Lekkerkerker, H. N. W. Insights into phase transition kinetics from colloid science. Nature 416, 811-815 (2002).

12. Cacciuto, A., Auer, S. \& Frenkel, D. Onset of heterogeneous crystal nucleation in colloidal suspensions. Nature 428, 404-406 (2004).

13. Oxtoby, D. W. Diversity suppresses growth. Nature 413, 694-695 (2001).

14. van Blaaderen, A., Ruel, R. \& Wiltzius, P. Template-directed colloidal crystallization. Nature 385, 321-324 (1997).

15. Dinsmore, A. D. \& Weitz, D. A. Direct imaging of three-dimensional structure and topology of colloidal gels. J. Phys. Condens. Matter 14, 7581-7597 (2002).

16. Heni, M. \& Löwen, H. Surface freezing on patterned substrates. Phys. Rev. Lett. 85, 3668-3671 (2000).

17. Nefedov, A. P. et al. PKE-Nefedov: plasma crystal experiments on the International Space Station. New J. Phys. 5, 33.1-33.10 (2003).

18. Landau, L. D. \& Lifshitz, E. M. Statistical Physics Part I (Pergamon, Oxford, 1978).

19. Zuzic, M. et al. Three-dimensional strongly-coupled plasma crystal under gravity conditions. Phys. Rev. Lett. 85, 4064-4067 (2000).

20. Flook, A. G. The use of dilation logic on the quantiment to achieve fractal dimension characterization of textured and structured profiles. Powder Technol. 21, 295-298 (1978).

21. Efron, B. \& Tibshirani, R. J. An Introduction to the Bootstrap (Chapman \& Hall, New York, 1993).

22. Konopka, U., Morfill, G. E. \& Ratke, L. Measurement of the interaction potential of microspheres in the sheath of a rf discharge. Phys. Rev. Lett. 84, 891-894 (2000).

\section{Acknowledgements}

Correspondence and requests for materials should be addressed to M.R.-Z.

\section{Competing financial interests}

The authors declare that they have no competing financial interests.

Reprints and permission information is available online at http://npg.nature.com/reprintsandpermissions/ 